\title{
Interactive effects of gender and sexual orientation on cortical thickness, surface area and gray matter volume: a structural brain MRI study
}

\author{
Dandan Wang ${ }^{1,2 \#}$, Lu Han ${ }^{3 \#}$, Caixi Xi ${ }^{1,2}$, Yi Xu ${ }^{1,2}$, Jianbo Lai ${ }^{1,2}$, Shaojia $\mathrm{Lu}^{1,2}$, Manli Huang ${ }^{1,2}$, Jianbo $\mathrm{Hu}^{1,2}$, \\ Ning Wei ${ }^{1,2}$, Weijuan $\mathrm{Xu}^{1,2}$, Weihua Zhou ${ }^{1,2}$, Qiaoqiao $\mathrm{Lu}^{1,2}$, Hongjian $\mathrm{He}^{3}$, Shaohua $\mathrm{Hu}^{1,2}$ \\ ${ }^{1}$ Department of Psychiatry, First Affiliated Hospital, Zhejiang University School of Medicine, Hangzhou 310003, China; ${ }^{2}$ The Key Laboratory of \\ Mental Disorder Management in Zhejiang Province, Hangzhou 310003, China; ${ }^{3}$ Center for Brain Imaging Science and Technology, Key Laboratory \\ for Biomedical Engineering of Ministry of Education, College of Biomedical Engineering and Instrumental Science, Zhejiang University, Hangzhou \\ 310027, China
}

"These authors contributed equally to this work.

Correspondence to: Shaohua Hu, MD. Department of Mental Health, First Affiliated Hospital, Zhejiang University School of Medicine, The Key Laboratory of Mental Disorder Management in Zhejiang Province, 79 Qing Chun Rd, Hangzhou 310003, China. Email: dorhushaohua@zju.edu.cn; Hongjian He, PhD. Center for Brain Imaging Science and Technology, Key Laboratory for Biomedical Engineering of Ministry of Education, College of Biomedical Engineering and Instrumental Science, Zhejiang University, Zhouyiqing Bldg., Yuquan Campus, Zhejiang University, Zheda Rd. 38, Hangzhou 310027, China. Email: hhezju@zju.edu.cn.

Background: Testosterone is thought to play a crucial role in sexual differentiation of the brain, and sexual orientation is programmed into our brain structures even when we are still fetuses. Although gender and sexual orientation differences have been shown respectively in many brain structures, the mechanism underlying the sexual differentiation of the brain is still unknown. The study is to investigate the interactive effects of gender and sexual orientation on cerebral structures in homosexual and heterosexual people.

Methods: Sexual orientation was evaluated by the Kinsey scale. We collected structural magnetic resonance image (MRI) data of local cortical thickness, surface area, and gray matter volume in all the subjects (29 homosexual and 29 heterosexual men, 17 homosexual and 17 heterosexual women). Statistical maps were generated using a general linear model (GLM) using FreeSurfer's Query, Design, Estimate, Contrast (QDEC) interface. We had sexual orientation and gender as 2 discrete factors with 2 levels, allowing for the generation of the interaction between sexual orientation and gender: homosexual women and heterosexual men versus heterosexual women and homosexual men. Coordinates were in Talairach space. All the cluster sizes were calculated with a $\mathrm{P}$ value of 0.01 .

Results: Results revealed interactions concerning the area and gray matter volume between the factors of sexual orientation and gender. Regarding the thickness, an interaction was not found in any regions of the clusters. Regarding the area, an interaction was found in region of left middle temporal lobe, inferior temporal lobe, lateral occipital lobe, fusiform $\left[(-58.1,-38.6,-14.7)\right.$, maximum vertex-wise $(\mathrm{MV}) \log _{10}(\mathrm{P})=3.30$, cluster size $(\mathrm{CS})=1,286.90 \mathrm{~mm}^{2}$ ], and left rostral middle frontal lobe, pars opercularis, caudal middle frontal lobe $\left[(-37.3,23.6,24.8), M V \log _{10}(\mathrm{P})=2.92, \mathrm{CS}=1,194.40 \mathrm{~mm}^{2}\right]$. Regarding the gray matter volume, an interaction was found in the region of the left pars opercularis (inferior frontal gyrus) $\left[(-42.9,6.3,18.5), M V \log _{10}(\mathrm{P})=1.31\right.$, $\left.\mathrm{CS}=526.79 \mathrm{~mm}^{2}\right]$.

Conclusions: The present study extends our understandings of how structural features differ in homosexual men, heterosexual men, homosexual women, and heterosexual women. Furthermore, it highlights the interactions between sexual orientation and gender in the left inferior frontal gyrus, bilateral temporal lobe, and the right rostral anterior cingulate cortex, which are suggested to play a critical role in the sexual differentiation of the human brain. 
Keywords: Interactive effects; structural magnetic resonance image (structural MRI); sexual orientation; gender

Submitted Jun 06, 2019. Accepted for publication Feb 28, 2020.

doi: 10.21037/qims.2020.03.07

View this article at: http://dx.doi.org/10.21037/qims.2020.03.07

\section{Introduction}

Testosterone is thought to play a crucial role in the sexual differentiation of the brain. As previous studies have shown, a surge of testosterone released in the embryo results in the brain developing in the male direction, while the absence of this surge feminizes the fetal brain (1). Testosterone is also believed to impact gender differences in local brain structures and some functions $(2,3)$, and also bears a potential link to sexual orientation (4).

Gender differences were initially found in the hypothalamus. More precisely, the dimorphic nucleus of the preoptic area (SDN-POA) was found to be bigger in males than in females (5). The other 2 important sexually dimorphic areas are also in the hypothalamus, and called the anteroventral periventricular nucleus (AVPV) and the ventromedial nucleus $(\mathrm{VMH})$; the former is larger in females than in males (6), with the reverse being the case in the latter (7). Structural differences have also been shown in other brain structures, including the human anterior commissure, the interthalamic adhesion, and the corpora mammillaria (8). Neuroimaging studies have provided evidence of gender differences in cortical thickness (9), regional gray and white matter volumes (4), and subcortical volumes (10). Resting-state functional magnetic resonance imaging (MRI) and diffusion tensor imaging (DTI) studies indicated that white matter microstructure and intrinsic neural networks differ between genders (11). Furthermore, in males, the amygdala showed greater activation than in females when the subjects viewed sexually stimulating pictures (12).

Sexual orientation related to structural and functional brain differences has also been reported extensively in the literature. The suprachiasmatic nucleus (SCN) is reported to be twice as large in homosexual men than in heterosexual men (13). Meanwhile, the interstitial nucleus of the anterior hypothalamus-3 (INAH-3) is considered to be a dimorphic area in sexual orientation, and is twice as large in heterosexual men than in homosexual men, whereas the volume of INAH-3 in heterosexual women has no difference compared with homosexual men (14).
Additionally, the anterior commissure and isthmus of the corpus callosum were reported to be larger in homosexual men than in heterosexual men (8). Resting-state functional MRI also demonstrate differences in functional connectivity (FC), regional homogeneity, and fractional amplitude of low-frequency fluctuation (fALFF) between homosexual and heterosexual men (15).

Moreover, an MRI study reported that the perirhinal cortex of homosexual female participants showed a more male-like structural pattern of gray matter (16). During sexual arousal, the hypothalamus is more weakly activated in homosexual men than in heterosexual men, and similar to the pattern displayed in heterosexual women (17). Safron et al. observed that activation of the amygdala was more pronounced in homosexual men than in heterosexual men (18), and the patterns of amygdala functional connection in homosexual men conformed with heterosexual women, while homosexual women showed more male-like features (19). What is worth nothing concerning the above studies is that the structural and functional differences in the cerebral area between homosexual and heterosexual men are common; nevertheless, some brain functions and local structures in homosexual men are always similar to those in heterosexual women. This point is confirmed by neurocognitive findings as well, which demonstrate the feminization of intelligence structures in homosexual men whose verbal intelligence quotient tests much better than the performance intelligence quotient in heterosexual men and shows less asymmetry (20).

These findings imply that gender and sexual orientation are both critical factors that are programmed into brain structures and functions along with sexual differentiation. Although considerable progress has been made in the understanding of the differences between genders and between sexual orientations acting on brain morphology and neural activity during sexual arousal, there have been only a small number of studies that have investigated the interaction between gender and sexual orientation. Thus, the current study aimed to investigate the interaction between sexual orientation and gender in a series of structural parameters, including cortical thickness, surface 
Table 1 Demographic and clinical characteristics for all subjects

\begin{tabular}{|c|c|c|c|c|c|}
\hline Variable & \multicolumn{2}{|c|}{ Men } & \multicolumn{2}{|c|}{ Women } & $P$ values \\
\hline $\mathrm{Age}^{\mathrm{a}}($ mean $\pm \mathrm{SD})$ & $23.17 \pm 2.75$ & $22.14 \pm 3.24$ & $24.00 \pm 2.48$ & $23.24 \pm 5.36$ & 0.35 \\
\hline \multicolumn{6}{|c|}{ Kinsey scale $(\mathrm{N}, \%)$} \\
\hline Score 0 & $29(100)$ & 0 & $17(100)$ & & \\
\hline Score 2 & & 0 & & & \\
\hline Score 3 & & $3(10.34)$ & & & \\
\hline Score 4 & & $7(24.14)$ & & & \\
\hline Score 5 & & $11(37.93)$ & & $10(58.82)$ & \\
\hline
\end{tabular}

${ }^{a}$, the $\mathrm{P}$ value was calculated using analyses of variance (ANOVAs); ${ }^{\mathrm{b}}$, all heterosexual subjects scored themselves as exclusively heterosexual, while none of the homosexual participants scored themselves as heterosexual with some scoring as bisexual and some scoring as homosexual. SD, standard deviation.

area, and gray matter volume.

\section{Methods}

\section{Subjects}

The present study recruited homosexual subjects from a local gay bar in Hangzhou, China, and the heterosexual subjects were recruited via advertisements posted in 2 local communities and Zhejiang University between 2012 and 2017. All subjects were pre-screened to ensure that they were between the ages of 18 and 35, right-handed, and had no earlier history of mental disorder or physical disease. We administered some routine examinations for the participants after the recruitment, including routine blood tests, blood biochemical examination, and electrocardiogram to guarantee that all subjects were in good condition. Furthermore, we performed a structured clinical interview, the Mini-International Neuropsychiatric Interview (MINI), for all subjects to exclude the possibility for other mental disorders. The sexuality of the homosexual subjects was assessed by the Kinsey Heterosexual-Homosexual rating scale, which ranges from 0 (exclusively heterosexual) to 6 (exclusively homosexual) during the initial interview at the gay bar, and the heterosexual subjects were assessed at the institution during the first interview. Scores for all heterosexual subjects were 0 , and those for the subjects with an originally homosexual orientation ranged from 3 to 6 (Table 1). Participants with a history of sexual dysfunction, gender dysphoria, paraphilia, or sexual offenses were also excluded from the study. Eventually, there remained 29 homosexual men (mean age, 22.14 3.24 years) 29 heterosexual men (mean age, $23.17 \pm 2.75$ years) groups, 17 heterosexual women (mean age, 24.00 \pm 2.48 years), and 17 homosexual women (mean age, $23.24 \pm 5.36$ years) as study subjects (Table 1). A previous study suggested that at least 18 subjects for each group are needed to achieve at least $80 \%$ power when limitations from budget and values of the mean and variance are taken into consideration (21). The small sample sizes of heterosexual and homosexual women are a limitation of the present study. The study complied with the Declaration of Helsinki and approved by the ethics committee of the First Affiliated Hospital, Zhejiang University School of Medicine, and written informed consent was received from each participant before participation in the study.

\section{Image acquisition}

MR images were collected on a 3.0-Tesla General Electric (GE) SIGNA Scanner with a standard GE whole-head coil. Participants lay on the scanner with foam cushions under the head to minimize head motion and with earplugs to reduce the noise. Whole-brain high-resolution 3D anatomical data were acquired by using a T1-weighted fast spoiled gradient-echo (Fast SPGR) sequence, with the following parameters: $\mathrm{TR}=7 \mathrm{~ms}, \mathrm{TE}=2.9 \mathrm{~ms}$, field 
of view $=240 \times 240 \mathrm{~mm}^{2}$, matrix size $=256 \times 256$, in-plane resolution $=0.9375 \times 0.9375 \mathrm{~mm}$, slice thickness $=1 \mathrm{~mm}$, and 170 interleaved axial slices in total without inter-slice gap. Imaging data in the study were mostly acquired in the evening, and all the participants were normally fed just before the MRI scanning. Participants were asked to abstain from drinking coffee or any other drink or drug that could affect brain function. The scanning sessions lasted 4 minutes and 52 seconds, and during the scanning process, all subjects were asked to keep their eyes closed, but to avoid falling asleep and thinking systematically.

\section{Image preprocessing}

Structural MRI data were analyzed to reconstruct geometrically accurate surface models using the reconall processing in FreeSurfer $(22,23)$ software version 5.3.0 (Massachusetts General Hospital, Harvard Medical School; (http://www.surfer.nmr.mgh.harvard.edu/). The fully automated pipeline started with Talairach transformation to the MNI305 atlas and correction for intensity bias, contributing to intensity-based segmentation (24). Skull striping was followed to remove the non-brain parts, and subcortical structures were labeled. White matter was isolated using intensity, neighborhood, and smoothness as constraints and was subsequently separated into 2 hemispheres based on the Voronoi diagram. The original surface could be created by covering each hemisphere with triangles, whose intersection points determined the vertices. After tessellation, topology correction and surface deformation were performed to optimally create the white matter surface following the T1 white-gray intensity gradients and the pial surface following the T1 graycerebro-spinal fluid (CSF) intensity gradients, while vertex identity was preserved. It laid the basis for anatomical measurements: the cortical thickness was calculated as the closest distance between the gray-white matter boundary surface and pial surface at each voxel on the tessellated surface (25); the surface area was defined as the average area of the triangles of which the vertex is a member, and the gray matter volume (26) was generated by the elementwise product of surface area and the cortical thickness for each vertex. Upon completion of the cortical models, the individual original surface was registered to the manual labeling spherical atlas based on individual cortical folding patterns to label appropriate regions on the cortical surface across subjects. Thus, $3 \mathrm{~d}$ cortical surface models containing localization information and corresponding cortical thickness, surface area, or gray matter volume measures existed for both hemispheres of each participant. All the surface reconstruction results reported in this paper were generated using the fully automated processing pipeline, and no manual intervention was involved $(27,28)$.

\section{Statistical analyses}

Cortex analyses were conducted to explore local cortical thickness, surface area, and gray matter volume differences in homosexual women, heterosexual women, homosexual men, and heterosexual men. Statistical maps that showed the distribution of $p$ values were generated by means of a general linear model (GLM) approach using FreeSurfer's Query, Design, Estimate, Contrast (QDEC) interface. QDEC is a single-binary application that is intended to calculate the average within a subject or between groups and make a statistical inference for error variance on the morphometric data obtained from the reconstruction Processing stream. It employed the different offsets, different slopes (DODS) method to automatically create a design matrix, meaning that each group had its linear model, which was plausible in this study. This factorial design had sexual orientation and gender as 2 discrete factors with 2 levels, allowing for the generation of the interaction between sexual orientation and gender: homosexual women and heterosexual men versus heterosexual women and homosexual men. For each hemisphere, analysis of cortical thickness, surface area, and gray matter volume were conducted, respectively, accounting for the interaction effects between sexual orientation and gender. Cortical maps were spatially smoothed by a $5 \mathrm{~mm}$ full width at half maximum (FWHM) Gaussian kernel. The results of significant cortical areas were laid on the semi-inflated cortical surfaces for better visualization. The statistical threshold was set at $\mathrm{P}=0.01$. Multiple comparisons of cortical thickness, surface area, and gray matter volume maps were corrected with a Monte Carlo Null-Z simulation using a threshold set at $\mathrm{P}=0.01$ and the sign set at positive.

\section{Region of interest}

The main analyses were followed up by analyses of exploring the effects of sexual orientation and gender on each significant different cluster produced by statistical analyses. The corresponding mean thickness, area, and volume data of each preceding statistical cluster were extracted from each participant and were then averaged 


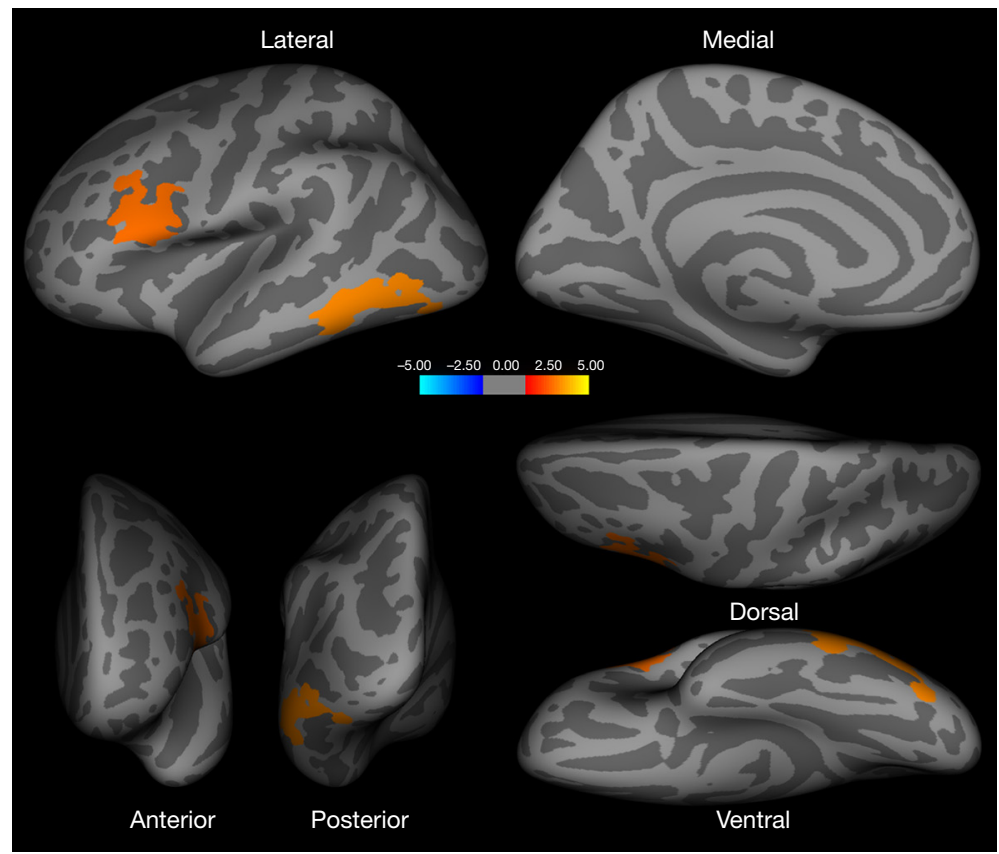

Figure 1 Brain regions showing statistically significant differences in surface area in the left hemisphere $(P<0.05, M o n t e ~ C a r l o ~ N u l l-Z$ simulation corrected). In the left middle and inferior temporal lobe, lateral occipital lobe, fusiform, pars opercularis, caudal and rostral MFG, and precentral, homosexual women and heterosexual men > heterosexual women and homosexual men. MFG, middle frontal gyrus.

according to grouping. Finally, there was a four-group average, representative of the 4 combined levels. Line graphs were used to detect evidence of the interaction between 2 factors: sexual orientation and gender. Lines that were not parallel within a graph represented an interaction, meaning that differences in one factor depended on the level of the other factor.

\section{Results}

\section{Cortical thickness analysis}

The statistical cortical thickness maps of both hemispheres did not have any findings that survived Monte Carlo Null-Z simulation correction. Homosexual women and heterosexual men did not show any region of thicker or thinner cortex than heterosexual women and homosexual men.

\section{Surface area analysis}

Homosexual women and heterosexual men showed a significantly larger surface area in the left middle and inferior temporal, lateral occipital, fusiform, pars opercularis [inferior frontal gyrus (IFG)], caudal and rostral middle frontal gyrus (MFG), and precentral gyrus (PCG) when compared with heterosexual women and homosexual men (Figure 1, Table 2). Additional regions of surface area increase were identified in the right inferior parietal, lateral occipital, middle and superior temporal, rostral anterior cingulate cortex (ACC), superior frontal gyrus (SFG), and medial orbitofrontal cortex (OFC) (Figure 2, Table 2). We then corrected the results listed above, with total intracranial volume and age as a covariate. The results suggest that significant interactive effects remained between gender and sexual orientation in these brain regions (Table 2). Furthermore, an interaction effect on the surface area of the cluster in the left and right hemispheres between 2 factors, sexual orientation and gender, was spotted by the intersection in (a-e) graphs of group means (Figure 3). The effects of sexual orientation in each ROI drawn from the 5 cluster regions (Table 2) measuring corresponding structural parameters depended on gender and vice versa.

\section{Gray matter volume analysis}

Increased gray matter volume was detected in the left pars opercularis [inferior frontal gyrus (IFG)], and, to some 
Table 2 Brain regions (surface area and gray matter volume) in homosexual women and heterosexual men that are significantly larger than those in homosexual men and heterosexual women

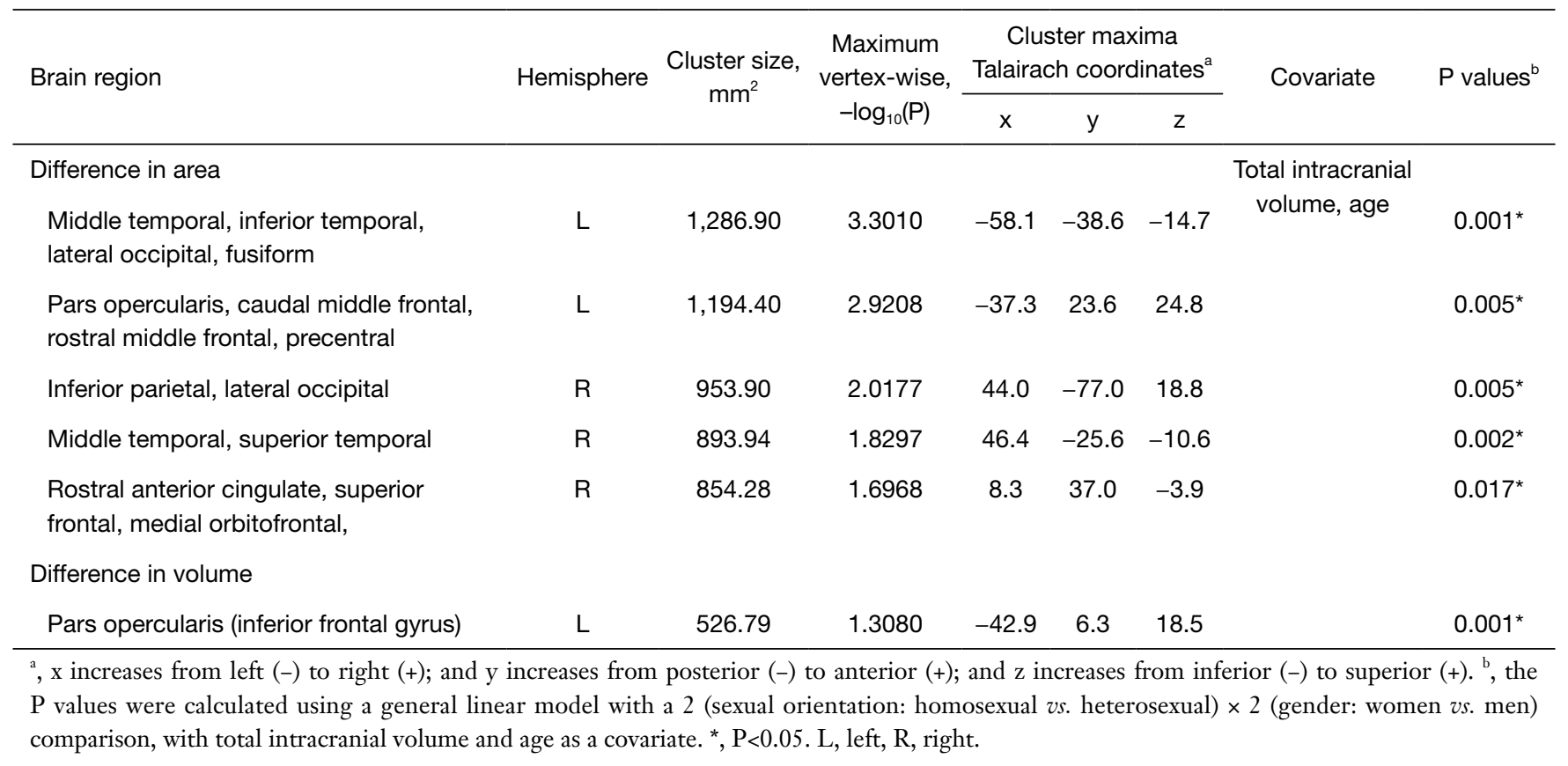



Figure 2 Brain regions showing statistically significant differences in surface area in the right hemisphere $(\mathrm{P}<0.05, M o n t e$ Carlo Null- $Z$ simulation corrected). In the right inferior parietal lobe, lateral occipital lobe, middle and superior temporal lobe, rostral ACC, SFG, and medial OFC, homosexual women and heterosexual men > heterosexual women and homosexual men. ACC, anterior cingulate cortex; SFG, superior frontal gyrus; OFC, orbitofrontal cortex. 
A Mean values of the four groups in \#1 cluster of area analysis $(\mathrm{L})$

1.00

0.80

0.60



\begin{tabular}{c|c|c}
\multirow{2}{*}{0.40} & & Male \\
\cline { 2 - 3 } & Female & 0.61 \\
\hline Homo & 0.65 & 0.69
\end{tabular}

C Mean values of the four groups in \#1 cluster of area analysis (R)

0.80

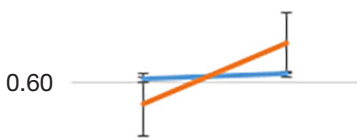

\begin{tabular}{c|c|c}
\multirow{2}{*}{0.40} & \multicolumn{2}{c}{ Male } \\
\cline { 2 - 3 } & Female & 0.61 \\
\hline Homo & 0.61 & 0.67
\end{tabular}

E

Mean values of the four groups in \#3 cluster of area analysis (R)

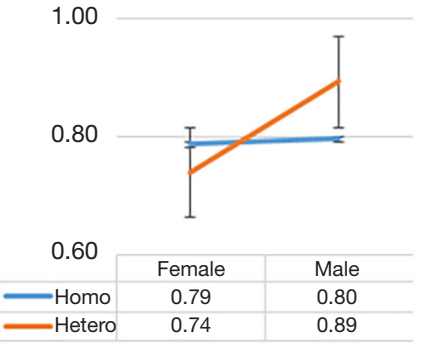

B Mean values of the four groups in \#2 cluster of area analysis (L)

1.00

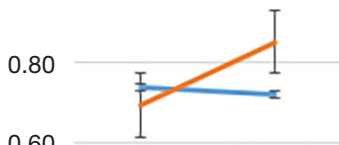

0.60

\begin{tabular}{|c|c|c|}
\cline { 2 - 3 } \multicolumn{1}{c|}{0.40} & Female & Male \\
\hline Homo & 0.74 & 0.72 \\
\hline Hetero & 0.69 & 0.85 \\
\hline
\end{tabular}

D Mean values of the four groups in \#2 cluster of area analysis (R) 0.80

0.60

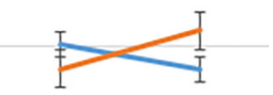

\begin{tabular}{|c|c|c|}
\hline 0.40 & Female & Male \\
\hline - Homo & 0.60 & 0.56 \\
\hline - Hetero & 0.56 & 0.63 \\
\hline
\end{tabular}

F Mean values of the four groups in \#1 cluster of volume analysis (L)

2.00

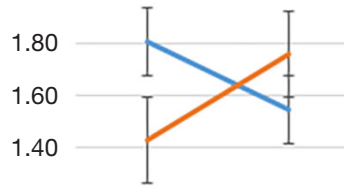

1.20

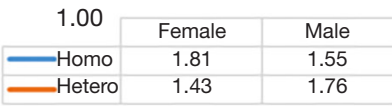

Figure 3 The intersection in every graph comparing group means among homosexual women, heterosexual women, heterosexual men, and homosexual men showing an interaction effect between two factors: sexual orientation and gender. The group means were calculated from the average surface area of (A) the first cluster and (B) the second cluster in the left hemisphere, and the average surface area of (C) the first cluster, (D) the second cluster, and (E) the third cluster in the right hemisphere, and the average volume of (F) the cluster in the left hemisphere (Table 2).

extent, aligned with the surface area analysis for the left hemisphere, in homosexual women and heterosexual men versus heterosexual women and homosexual men (Figure 4, Table 2). Next, we corrected the result with total intracranial volume and age as a covariate; significant interactive effects between gender and sexual orientation remained for the left pars opercularis (Table 2). Additionally, an interaction effect on the volume of the cluster in the left hemisphere between 2 factors, sexual orientation and gender, was spotted by the intersection in the (f) graph of group means (Figure 3). The effects of sexual orientation in each ROI drawn from the cluster region (Table 2) measuring corresponding structural parameters depended on gender and vice versa. There were no significant differences in the gray matter volume analysis of the right hemisphere.

\section{Discussion}

To our knowledge, this is the first study to investigate the interaction between sexual orientation and gender in a series of structural parameters, including cortical thickness, surface area, and gray matter volume. Results revealed interactions in respect to the area and gray matter volume between the factors of sexual orientation and gender, which 


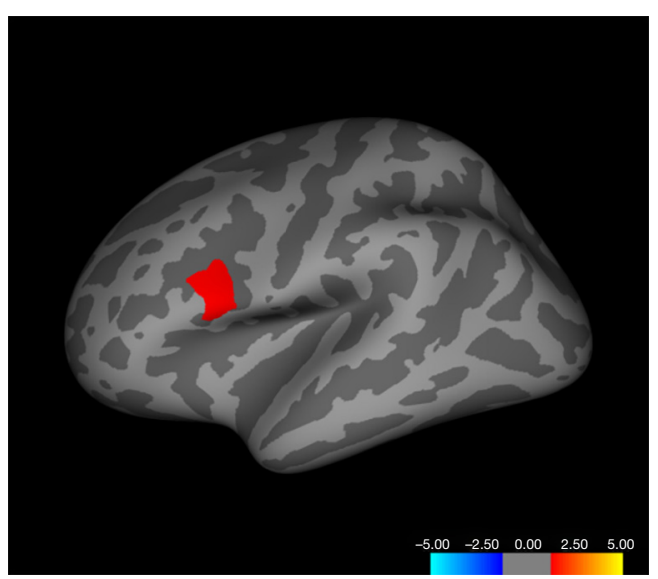

Figure 4 Brain regions showing statistically significant differences in gray matter volume in the left hemisphere $(\mathrm{P}<0.05$, Monte Carlo Null-Z simulation corrected). In the pars opercularis, Homosexual women and heterosexual men $>$ heterosexual women and homosexual men.

supported the notion that homosexual men and heterosexual women display a similar pattern in certain regions of brain structure. We were able to demonstrate that these brain structures might be the region with the interaction between gender and sexual orientation. As listed above, homosexual men and heterosexual women showed no difference in the cortical thickness of visual areas (29).

As for our study, homosexual women and heterosexual men were not significantly different from heterosexual women and homosexual men regarding cortical thickness. Further, homosexual women and heterosexual men showed a markedly larger surface area than heterosexual women and homosexual men in the left temporal, IFG, MFG, lateral occipital, fusiform, precentral and right inferior parietal, lateral occipital, middle and superior temporal, rostral ACC, SFG, and medial OFC. Regarding gray matter volume, the left pars opercularis of IFG was found to be larger in homosexual women and heterosexual men versus heterosexual women and homosexual men. These findings imply a within-interaction between gender and sexual orientation in the function of the frontal, occipital, parietal, and temporal cortices. The result that homosexual women and heterosexual men showed larger surface areas in both hemispheres is consistent with a previous study which revealed a larger surface area bilaterally in medial occipital cortices compared with heterosexual women (30), suggesting a more "male-typical" pattern among homosexual women. This "male greater than female" pattern of cortical volume in medial occipital brain regions has also been reported in a recent meta-analysis (31). Previous evidence has pointed out that the intelligence structure of male homosexuals approached that of female heterosexuals, who performed much better than heterosexual men in semantic monitoring tasks, lexical decision-making, and mental rotation (20). This interaction in the temporal lobe, which is a key region for memory and semantic judgment (32) is consistent with our findings. However, the larger surface area in most of the temporal lobe bilaterally in homosexual women and heterosexual men of our study disagreed with its inferiority in the verbal and spatial ability.

Nevertheless, with the neuropsychological tests, other later studies have revealed that heterosexual men performed better in visuospatial ability than homosexual men and heterosexual women, while the performances of the latter 2 did not differ significantly (33). What is more, homosexual men displayed female-like performance on mental rotation items, and the performance was worse than that of heterosexual men (34), which corroborated our findings from the side. Varying cognitive measures and outcome bias may account for the discrepant results here.

In contrast, some studies reported that homosexual men and heterosexual women both outperformed heterosexual men and homosexual women in category fluency, and homosexual men outperformed other groups in letter and synonym fluency (33). The verbal fluency test is a certain neurocognitive test for executive function (35). The prefrontal cortex is thought to be a key region for executive function, which is defined as higher-order cognitive abilities, including the process of attention and inhibition, task management, planning, monitoring, and coding (36). Other frontal clusters, parietal structures, and the anterior cingulate, have also been reported to be critical to executive function (37). In particular, research has shown that the MFG is part of an executive attention network (38), modulates executive function and attention processes (39), and is further related to inhibitory control/motor impulsiveness (40). Additionally, the cortical thickness of the bilateral MFG had been described as being positively correlated with cognitive manipulation (41).

Moreover, the gray matter volume reduction of the bilateral OFC was observed in depressive patients with suicide attempts relating to impulsiveness compared to controls (42). MFG is the key region for top-down control, and together with MFG, the OFC and SFG is understood as involved in several aspects of impulsiveness (37). The left IFG has been suggested to play a role in controlling behavioral inhibition, including the tendency 
to inhibit learning from undesirable information (43). Inconsistent with the evidence mentioned above is that the present results suggest the superiority of homosexual women and heterosexual men in the surface area and gray matter volumes in the cognition-related brain structure. However, our previous reported a decreased fALFF in the left IFG in homosexual men compared with heterosexual participants (15). Furthermore, one of our currently pending studies showed that homosexual men performed poorer in the Wisconsin Card Sorting Test (WCST) and partial CPT measurements than heterosexual participants, which is considered as a measure of executive function because of its reported sensitivity to frontal lobe dysfunction (44). In line with the studies mentioned above, the present results suggest an interaction in surface area of the left IFG, right SFG, left MFG, right OFC, parietal and right ACC, and gray matter volume of the left IFG; larger surface area and gray matter volumes in the above regions were displayed in homosexual women and heterosexual men. The inconsistency with other groups may be ascribed to 2 mechanisms. One is the deficiency of the studies related to the sexual orientation difference in cognitive function, which leads to the great heterogeneity of the results. Another is the methodological difference between MRI imaging processing and acquisition parameters. On the other hand, there is a possibility that there is no specific functional association with the change of brain structural parameters of the regional cortex (e.g., cortical thickness, surface area, gray matter volume), although studies show a relationship between brain structural parameters and function theoretically (29). There is still developing yet conflicting evidence for the influence of sexual orientation on sexually dimorphic cognitive abilities.

Gender and sexual orientation are both considered to be shaped into brain structures and functions along with the sexual differentiation; however, the neural basis underlying sexual differentiation is still unclear. It is widely acknowledged that testosterone is capable of mediating sexual differentiation of brain structures, both during the prenatal period and in puberty (45), and testosteronedependent changes in gray matter and cortical thickness across puberty in several regions involved in cognitive control, and emotional regulation have been reported (46). There is evidence showing that testosterone levels tend to be negatively correlated with cortical thickness during adolescence, and that lower testosterone is associated with neuroprotective effects and increased cortical thickness (47), with inverse correlations being reported between parietal and occipital cortical thickness and testosterone levels (9). For gray matter volumes, sexually mature boys exhibit a larger volume of medial temporal lobe structure volume than sexually mature girls (48), while another study revealed that testosterone levels were inversely associated with gray matter volume in the ACC and OFC with sex-specific differences (49). The theory that male homosexuality correlates to a lack of testosterone when the embryo is in utero, while an excess release of testosterone to a female fetus leads to female homosexuality (50) is not supported by our findings which show larger surface area and gray matter volumes in homosexual women and heterosexual men in the occipital, parietal, ACC, OFC, temporal lobe; a possible reason for this may be the subsequent postnatal impact of testosterone particularly across puberty. Another study explored the influence of two-year testosterone replacement therapy on cognition and found that androgen treatment promoted an enhancement in attention and visual scanning ability, executive function, and psychomotor speed (51) while other research has demonstrated that testosterone has an impact on visuospatial processing in humans (52). Moreover, androgen deprivation in rodents by gonadectomy was shown to impair learning, memory, and executive function, while testosterone replacement normalized the performance (53). The above-mentioned testosteronemediated cognition-related regions are areas where an interaction between gender and sexual orientation exists, and likely have a critical role in the sexual differentiation of the human brain.

\section{Conclusions}

In conclusion, the present study suggests a larger surface area and gray matter volume in some specific cerebral areas in homosexual women and heterosexual men versus homosexual men and heterosexual women. Our findings extend our understanding of how structural features differ between homosexual men, heterosexual men, homosexual women, and heterosexual women, and highlight the interaction between sexual orientation and gender in the left IFG, MFG, and SFG, and the right ACC, bilateral temporal lobe, bilaterally lateral occipital lobe and other areas. It appears that they are critically involved in the sexual differentiation of the human brain. A specific link between testosterone, gender, and sexual orientation, brain structure, and function in the developing brain has yet to be fully explored, and may be the key to revealing the underlying neural basis of sexual differentiation of the brain. 


\section{Acknowledgments}

Funding: This work was supported by the National Natural Science Foundation of China (NSFC) (No. 81671357 and 81771474), and the grants of the Public Welfare Project of Science Technology Department of Zhejiang Province (No. 2015C33133).

\section{Footnote}

Conflicts of Interest: All authors have completed the ICMJE uniform disclosure form (available at http://dx.doi. org/10.21037/qims.2020.03.07). The authors have no conflicts of interest to declare.

Ethical Statement: The study complied with the Declaration of Helsinki and approved by the ethics committee of the First Affiliated Hospital, Zhejiang University School of Medicine, and written informed consent was received from each participant before participation in the study.

Open Access Statement: This is an Open Access article distributed in accordance with the Creative Commons Attribution-NonCommercial-NoDerivs 4.0 International License (CC BY-NC-ND 4.0), which permits the noncommercial replication and distribution of the article with the strict proviso that no changes or edits are made and the original work is properly cited (including links to both the formal publication through the relevant DOI and the license). See: https://creativecommons.org/licenses/by-nc-nd/4.0/.

\section{References}

1. Bao AM, Swaab DF. Sexual differentiation of the human brain: relation to gender identity, sexual orientation and neuropsychiatric disorders. Front Neuroendocrinol 2011;32:214-26.

2. Höfer P, Lanzenberger R, Kasper S. Testosterone in the brain: neuroimaging findings and the potential role for neuropsychopharmacology. Eur Neuropsychopharmacol 2013;23:79-88.

3. Savic I, Frisen L, Manzouri A, Nordenstrom A, Linden Hirschberg A. Role of testosterone and Y chromosome genes for the masculinization of the human brain. Hum Brain Mapp 2017;38:1801-1814.

4. Lentini E, Kasahara M, Arver S, Savic I. Sex differences in the human brain and the impact of sex chromosomes and sex hormones. Cereb Cortex 2013;23:2322-36.
5. Swaab DF, Fliers E. A sexually dimorphic nucleus in the human brain. Science 1985;228:1112-5.

6. Bleier R, Byne W, Siggelkow I. Cytoarchitectonic sexual dimorphisms of the medial preoptic and anterior hypothalamic areas in guinea pig, rat, hamster, and mouse. J Comp Neurol 1982;212:118-30.

7. Dörner G, Staudt J. Structural changes in the hypothalamic ventromedial nucleus of the male rat, following neonatal castration and androgen treatment. Neuroendocrinology 1969;4:278-81.

8. Allen LS, Gorski RA. Sexual dimorphism of the anterior commissure and massa intermedia of the human brain. J Comp Neurol 1991;312:97-104.

9. Savic I, Arver S. Sex differences in cortical thickness and their possible genetic and sex hormonal underpinnings. Cereb Cortex 2014;24:3246-57.

10. Neufang S, Specht K, Hausmann M, Gunturkun O, Herpertz-Dahlmann B, Fink GR, Konrad K. Sex differences and the impact of steroid hormones on the developing human brain. Cereb Cortex 2009;19:464-73.

11. Filippi M, Valsasina P, Misci P, Falini A, Comi G, Rocca MA. The organization of intrinsic brain activity differs between genders: a resting-state fMRI study in a large cohort of young healthy subjects. Hum Brain Mapp 2013;34:1330-43.

12. Hamann S, Herman RA, Nolan CL, Wallen K. Men and women differ in amygdala response to visual sexual stimuli. Nat Neurosci 2004;7:411-6.

13. Swaab DF, Hofman MA. An enlarged suprachiasmatic nucleus in homosexual men. Brain Res 1990;537:141-8.

14. LeVay S. A difference in hypothalamic structure between heterosexual and homosexual men. Science 1991;253:1034-7.

15. Hu S, Xu D, Peterson BS, Wang Q, Lai J, Hu J, Wei N, Zhang $M, X u$ Y. Differing default mode network activities in men with homosexual or heterosexual preferences. J Sex Med 2014;11:2474-84.

16. Ponseti J, Siebner HR, Kloppel S, Wolff S, Granert O, Jansen O, Mehdorn HM, Bosinski HA. Homosexual women have less grey matter in perirhinal cortex than heterosexual women. PLoS One 2007;2:e762.

17. Paul T, Schiffer B, Zwarg T, Kruger TH, Karama S, Schedlowski M, Forsting M, Gizewski ER. Brain response to visual sexual stimuli in heterosexual and homosexual males. Hum Brain Mapp 2008;29:726-35.

18. Safron A, Barch B, Bailey JM, Gitelman DR, Parrish TB, Reber PJ. Neural correlates of sexual arousal in homosexual and heterosexual men. Behav Neurosci 
2007;121:237-48.

19. Savic I, Lindstrom P. PET and MRI show differences in cerebral asymmetry and functional connectivity between homo- and heterosexual subjects. Proc Natl Acad Sci U S A 2008; 105:9403-8.

20. Sanders G, Wright M. Sexual orientation differences in cerebral asymmetry and in the performance of sexually dimorphic cognitive and motor tasks. Arch Sex Behav 1997;26:463-80.

21. Mumford JA, Nichols TE. Power Calculation for Group fMRI Studies Accounting for Arbitrary Design and Temporal Autocorrelation. Neuroimage 2008;39:261-8.

22. Fischl B. FreeSurfer. Neuroimage 2012;62:774-81.

23. Zheng F, Li C, Zhang D, Cui D, Wang Z, Qiu J. Study on the sub-regions volume of hippocampus and amygdala in schizophrenia. Quant Imaging Med Surg 2019;9:1025-36.

24. Fischl B, Salat DH, van der Kouwe AJ, Makris N, Segonne F, Quinn BT, Dale AM. Sequence-independent segmentation of magnetic resonance images. Neuroimage 2004;23 Suppl 1:S69-84.

25. Fischl B, Dale AM. Measuring the thickness of the human cerebral cortex from magnetic resonance images. Proc Natl Acad Sci U S A 2000;97:11050-5.

26. Greve DN, Van der Haegen L, Cai Q, Stufflebeam S, Sabuncu MR, Fischl B, Brysbaert M. A surface-based analysis of language lateralization and cortical asymmetry. J Cogn Neurosci 2013;25:1477-92.

27. Dale AM, Fischl B, Sereno MI. Cortical surface-based analysis. I. Segmentation and surface reconstruction. Neuroimage 1999;9:179-94.

28. Fischl B, Sereno MI, Dale AM. Cortical surface-based analysis. II: Inflation, flattening, and a surface-based coordinate system. Neuroimage 1999;9:195-207.

29. Abé C, Johansson E, Allzen E, Savic I. Sexual orientation related differences in cortical thickness in male individuals. PLoS One 2014;9:e114721.

30. Abé C, Rahman Q, Langstrom N, Ryden E, Ingvar M, Landen M. Cortical brain structure and sexual orientation in adult females with bipolar disorder or attention deficit hyperactivity disorder. Brain Behav 2018;8:e00998.

31. Ruigrok AN, Salimi-Khorshidi G, Lai MC, Baron-Cohen S, Lombardo MV, Tait RJ, Suckling J. A meta-analysis of sex differences in human brain structure. Neurosci Biobehav Rev 2014;39:34-50.

32. Vandenberghe R, Price C, Wise R, Josephs O, Frackowiak RS. Functional anatomy of a common semantic system for words and pictures. Nature 1996;383:254-6.

33. Rahman Q, Abrahams S, Wilson GD. Sexual-orientation- related differences in verbal fluency. Neuropsychology 2003;17:240-6.

34. Rahman Q, Andersson D, Govier E. A specific sexual orientation-related difference in navigation strategy. Behav Neurosci 2005;119:311-6.

35. Godard J, Baruch P, Grondin S, Lafleur MF. Psychosocial and neurocognitive functioning in unipolar and bipolar depression: a 12-month prospective study. Psychiatry Res 2012;196:145-53.

36. Smith EE, Jonides J. Storage and executive processes in the frontal lobes. Science 1999;283:1657-61.

37. Schilling C, Kuhn S, Romanowski A, Schubert F, Kathmann N, Gallinat J. Cortical thickness correlates with impulsiveness in healthy adults. Neuroimage 2012;59:824-30.

38. Andersson M, Ystad M, Lundervold A, Lundervold AJ. Correlations between measures of executive attention and cortical thickness of left posterior middle frontal gyrus - a dichotic listening study. Behav Brain Funct 2009;5:41.

39. Solanto MV, Schulz KP, Fan J, Tang CY, Newcorn JH. Event-related FMRI of inhibitory control in the predominantly inattentive and combined subtypes of ADHD. J Neuroimaging 2009;19:205-12.

40. Simmonds DJ, Pekar JJ, Mostofsky SH. Meta-analysis of Go/No-go tasks demonstrating that fMRI activation associated with response inhibition is task-dependent. Neuropsychologia 2008;46:224-32.

41. Ehrlich S, Brauns S, Yendiki A, Ho BC, Calhoun V, Schulz SC, Gollub RL, Sponheim SR. Associations of cortical thickness and cognition in patients with schizophrenia and healthy controls. Schizophr Bull 2012;38:1050-62.

42. Monkul ES, Hatch JP, Nicoletti MA, Spence S, Brambilla P, Lacerda AL, Sassi RB, Mallinger AG, Keshavan MS, Soares JC. Fronto-limbic brain structures in suicidal and non-suicidal female patients with major depressive disorder. Mol Psychiatry 2007;12:360-6.

43. Goghari VM, MacDonald AW 3rd. The neural basis of cognitive control: response selection and inhibition. Brain Cogn 2009;71:72-83.

44. Goldberg E, Bougakov D. Neuropsychologic assessment of frontal lobe dysfunction. Psychiatr Clin North Am 2005;28:567-80, 578-569.

45. Peper JS, Hulshoff Pol HE, Crone EA, van Honk J. Sex steroids and brain structure in pubertal boys and girls: a mini-review of neuroimaging studies. Neuroscience 2011;191:28-37.

46. Nguyen TV, McCracken JT, Ducharme S, Cropp BF, Botteron KN, Evans AC, Karama S. Interactive effects 
of dehydroepiandrosterone and testosterone on cortical thickness during early brain development. J Neurosci 2013;33:10840-8.

47. Nguyen TV, McCracken J, Ducharme S, Botteron KN, Mahabir M, Johnson W, Israel M, Evans AC, Karama S, Brain Development Cooperative G. Testosterone-related cortical maturation across childhood and adolescence. Cereb Cortex 2013;23:1424-32.

48. Bramen JE, Hranilovich JA, Dahl RE, Forbes EE, Chen J, Toga AW, Dinov ID, Worthman CM, Sowell ER. Puberty influences medial temporal lobe and cortical gray matter maturation differently in boys than girls matched for sexual maturity. Cereb Cortex 2011;21:636-46.

49. Koolschijn PC, Peper JS, Crone EA. The influence of sex steroids on structural brain maturation in adolescence. PLoS One 2014;9:e83929.

Cite this article as: Wang D, Han L, Xi C, Xu Y, Lai J, Lu S, Huang M, Hu J, Wei N, Xu W, Zhou W, Lu Q, He H, Hu S. Interactive effects of gender and sexual orientation on cortical thickness, surface area and gray matter volume: a structural brain MRI study. Quant Imaging Med Surg 2020;10(4):835-846. doi: 10.21037/qims.2020.03.07
50. Bailey JM. The man who would be queen. Washington DC: Joseph Henry Press, 2003.

51. Lašaitė L, Čeponis J, Preikša RT, Žilaitienė B. Effects of two-year testosterone replacement therapy on cognition, emotions and quality of life in young and middle-aged hypogonadal men. Andrologia 2017. doi: 10.1111/ and. 12633.

52. Goetz SM, Tang L, Thomason ME, Diamond MP, Hariri AR, Carré JM. Testosterone rapidly increases neural reactivity to threat in healthy men: A novel twostep pharmacological challenge paradigm. Biol Psychiatry 2014;76:324-31.

53. Edinger KL, Frye CA. Testosterone's analgesic, anxiolytic, and cognitive-enhancing effects may be due in part to actions of its 5alpha-reduced metabolites in the hippocampus. Behav Neurosci 2004;118:1352-64. 